\title{
Profile of Neonatal sepsis
}

Y. R. Khinchi ${ }^{1}$, Anit Kumar ${ }^{2}$, Satish Yadav ${ }^{3}$

${ }^{1}$ Associate Professor, ${ }^{2}$ Second year MD resident, ${ }^{3}$ Third year MD resident; Department of Pediatrics and Neonatology, College of Medical Sciences, Bharatpur, Nepal

\begin{abstract}
Objective: To study the clinical presentation, investigational profile and outcome of neonatal sepsis in general and with special reference to inborn (intramural) or out born (extramural), sex and weight of the neonate.

Materials and method: Retrospective descriptive study of neonates admitted during 2 years from July 2007 to June 2009 in special care neonatal unit of the department of Pediatrics, College of Medical Sciences-Teaching Hospital, Bharatpur, Nepal.
\end{abstract}

Results: Majority of neonates were out born and referred (72.2\%) to this institution. Significant number of babies was having sepsis in out born group (59\%) as compared to inborn (35\%). Male sex was found to have more sepsis as compared to female. Sepsis was observed to be inversely related to birth weight, $65 \%$ in low birth weight (LBW, $<2.5 \mathrm{Kg}$ ) as compared to $42.6 \%$ in normal birth weight group $(>2.5 \mathrm{~kg}$ ).

Conclusions: High index of suspicion for diagnosis of neonatal sepsis is required specially in the presence of risk factors. Prevalence of sepsis is inversely related to birth weight. More number of out born delivered babies developed sepsis. Neonatal sepsis related morbidity and mortality can be significantly reduced by comprehensive obstetric and neonatal care at multiple levels right from community to health institutions.

Key words: Neonatal sepsis, Inborn, Out born, Low birth weight (LBW).

\section{Introduction}

Neonatal sepsis is the single most important cause of neonatal deaths in the community, accounting for half of them. If diagnosed early and treated aggressively with antibiotics and good supportive care, it may be possible to save most cases of neonatal sepsis ${ }^{1}$. Surviving infants can have significant neurological

Correspondence: Dr. Y. R.Khinchi

E-mail:dr_khinchi@yahoo.com sequelae as a consequence of CNS involvement, septic shock or hypoxemia secondary to severe parenchymal lung disease ${ }^{2}$.

Neonatal sepsis is defined as a clinical syndrome of bacteremia with signs and symptoms of infection in the first four weeks of life. When pathogenic bacteria gain access into the blood stream, they may cause overwhelming infection without much localization 
termed as septicemia or may get predominantly localized to the lungs resulting in pneumonia, or the meninges causing meningitis. Early onset and late onset sepsis are defined on the basis of presentation within 72 hours or after 72 hours of life respectively.

There are many risk factors for development of neonatal sepsis including low birth weight, unsafe place of delivery or unclean delivery, prolonged rupture of membranes more than 24 hours, maternal pyrexia, chorio-amnionitis, prolonged labor and perinatal asphyxia.

The present study is carried out to determine the clinicoinvestigational profile and outcome of neonatal sepsis in general and specially in relation to place of delivery whether inborn or out born, sex, and weight of neonates admitted in special neonatal care unit of this institution.

\section{Materials and methods}

This study included newborns admitted in special care neonatal unit of college of medical sciences teaching hospital, Bharatpur, Nepal from July 2007 to June 2009.

This is a retrospective descriptive study. The case records of 411 newborns entitled for the study during this period was evaluated and categorized on the basis of septic or non septic; inborn (intramural: delivered in the study institution) or out born (extramural: delivered in other heath facility or home); sex and birth weight. The case records of these newborns was thoroughly studied and recorded for relevant information including history, clinical evaluation and available investigation either favoring primary diagnosis of neonatal sepsis including with co-morbid conditions or having some other exclusive alternate diagnosis other than sepsis.
The diagnosis of neonatal sepsis was based on clinical profile and septic screen, X-ray chest and or blood culture whenever feasible and ruling out any other exclusive alternate diagnosis. Neonates diagnosed as having neonatal sepsis on the basis of clinicoinvestigational evaluation with co-morbid conditions were also included as septic cases in the study.

Neonates taken against medical advice (LAMA) or referred or taken elsewhere on request or discharged on request before proper diagnosis were excluded from the study. All other neonates not fulfilling the diagnosis of sepsis as mentioned above were considered as having other alternate diagnosis and put in the category of non-septic cases. Statistical analysis was done by evaluating $p$ value.

\section{Results}

Total number of cases enrolled for the study was 411 which included 271 male and 140 female patients. There were 297 out born and 114 inborn neonates. Clinical and investigational profile of inborn and out born neonates is shown in the table-1. Septic group included 215 patients as compared to 196 in non-septic group (table-2). Neonatal sepsis accounted for 175 $(59 \%)$ in the out born and $40(35 \%)$ in the inborn category $(\mathrm{p}<0.05$, table- 3$)$. There was significant difference for prevalence of sepsis between male $(65.1 \%)$ and female $(34.9 \%)$ neonates $(\mathrm{p}<0.05$, table2). Early onset sepsis was responsible for 131 cases whereas late onset sepsis was observed in 84 neonates $(p<0.05$, table-4). Sepsis was significantly $(\mathrm{p}<.000$, table-5) much higher (69.7\%) in very low birth weight/ extremely low birth weight $(<1.5 \mathrm{Kg})$, followed by $(64.3 \%)$ in LBW $(<2.5 \mathrm{~kg})$ than normal birth weight $(>2.5 \mathrm{Kg})$ babies $(42.6 \%)$. 
Y. R. Khinchi et al, Profile of Neonatal sepsis

\section{Discussion}

Sepsis is the commonest cause of neonatal morbidity and mortality. It is responsible for about $30-50 \%$ of total neonatal deaths ${ }^{3,4}$. Sepsis related morbidity and mortality is largely either preventable or treatable with rational antimicrobial and supportive therapy. $\mathrm{LBW}$ is a strong risk factor for neonatal sepsis due to multiple reasons. Unsafe delivery or unclean delivery at inappropriate place is another important predisposing factor for sepsis.

Earliest clinical features of neonatal sepsis are often subtle and non specific therefore a high index of suspicion is needed for early diagnosis specially so if risk factors are also present. In the present study majority of neonates presented (table-1) with refusal to feeds $(74 \%)$, tachypnea or respiratory distress $(75 \%)$ and fever (69\%). In a study done in the tertiary care center in Bangladesh poor feeding, respiratory distress and fever was reported in $22.2 \%, 27.8 \%$ and $44.4 \%$ cases respectively ${ }^{5}$. In the same study they documented hypothermia in $11.1 \%$, apnea in $16.7 \%$, cyanosis in $11.1 \%$, convulsions in $11.1 \%$ and jaundice in $50 \%$ as compared to our findings $11.6 \%, 15 \%, 41 \%$, $20.4 \%$, and $40 \%$ respectively. Clinical features and further course in neonatal sepsis depends on various factors like birth weight, place of delivery, age of newborn, intervention in preventable factors for sepsis, availability, accessibility, affordability and timely referral of baby to an appropriate center. Therefore variation in different parameters may be observed in various studies.

Though the gold standard for the diagnosis of sepsis is positive blood culture but in our setup due to limitation of affordability, limited blood sample from newborn, prior use of antibiotics before admission and other factors, diagnosis is mainly clinical supported by septic screen and X-Ray chest. CSF examination and neuroimaging or other relevant investigations were done whenever clinically indicated.

Neonatal sepsis was diagnosed in $52.6 \%$ of neonates during the previous study done from January 06 to June 07 in this institution which is comparable to present data $(52.3 \%)$, reflecting that there is no change in factors having impact on prevention of neonatal sepsis in this region at all levels right from community to health facilities ${ }^{6}$.

Male neonates were reported to be affected more with sepsis as compared to females in some studies ${ }^{7,8}$. This is in concordance with our study as well $(\mathrm{p}<0.05$, table-2). Bias for male sex, place of study, sample including other factors may be responsible for increased number of male cases in these studies.

There was statistically significant difference ( $<<0.05$, table-3) in sepsis cases born in the study institution (inborn) as compared to those brought from outside (out born). In Inborn category 35\% had sepsis as compared to $59 \%$ in out born group. This is because of the fact that intramural deliveries in the study institution are conducted with preventive aspects with adequate perinatal care whereas in extramural including home deliveries there may be various predisposing and risk factors including unsafe or unclean environment, limited skilled manpower and inadequate facilities etc.

Early onset sepsis was documented significantly more as compared to late onset sepsis $(\mathrm{p}<0.05$, table4). Early onset neonatal sepsis in general is more common because of various high risk perinatal factors for sepsis operate during this period.

LBW is a strong risk factor contributing to sepsis. In this study birth weight is inversely related to 
development of sepsis which is statistically highly significant $(\mathrm{p}<0.000$, table- 4$)$. This is in concordance with other studies where low birth was found to be important risk factor for sepsis ${ }^{6,9}$. LBW babies are mostly also premature and are predisposed to sepsis due to multiple reasons like immune incompetence at various levels of defense, more subjected to invasive interventions etc.

Mortality due to sepsis in inborn was $7.5 \%$ as compared to $11 \%$ in out born with overall mortality of $10.2 \%$ (table-1) which is comparable to other hospital based study ${ }^{6}$. These data are low as compared to overall mortality which is reported to be in the range of $30-50 \%$ of total neonatal deaths in the community and rural India ${ }^{3,4}$. This wide difference in mortality may be due to the fact that our data are hospital based where we get selected referred out born babies and due to less neonatal sepsis in inborn neonates because deliveries are conducted with proper antenatal and perinatal services in this institution.

\section{Conclusions}

High index of suspicion for diagnosis of neonatal sepsis is required specially in the presence of risk factors and baby presenting with non specific clinical features. Prevalence of sepsis is inversely related to birth weight. More number of out born delivered cases develops sepsis due to lack of poor knowledge, accessibility, affordability or inadequate perinatal services in the community setup.

Neonatal sepsis related morbidity and mortality can be significantly reduced with good maternal nutrition and health status which is known to improve the birth weight of newborn along with encouraging institutional deliveries where adequate obstetric and neonatal services are available.

\section{References}

1. Management of neonatal sepsis, IAP-NNF (Indian Academy of Pediatrics- National Neonatology Forum) Guidelines 2006 on level II neonatal care: 159-86.

2. Chaiko B and Sohi I, Early onset neonatal sepsis. Indian Journal of Pediatrics, 72: 2005: 23-6.

3. Bang AT, Bang RA, Bactule SB et al. Effect of homebased neonatal care and management of sepsis on neonatal mortality: field trial in rural India. Lancet 1999 : 354: $1955-86$.

4. Stoll BJ, The global impact of neonatal infection. Clin Perinatol 1997: 24: 1-21.

5. Ahmed NU, Chowdhary A, Hoque M et al. Clinical and bacteriological profile of neonatal septicemia in a tertiary level pediatric hospital in Bangladesh. Indian Pediatrics 2002: 39: 1034-39.

6. Khinchi YR, Shreshta D, Sarmah BK et al. A study of morbidity and mortality profile of neonates admitted in tertiary care hospital in central Nepal. Journal of College of Medical Sciences, Nepal, 2008: 5: 70-5.

7. Jain NK, Jain VM, Maheshwari S. Clinical profile of neonatal sepsis. KUMJ: 2003: 1: 117-20.

8. WHO young infant study group. Bacterial etiology of serious infection in young infants in developing countries: results of multicenter study. Pediatr. Dis. J. 1999: 18 S17-22.

9. Jeeva Sankar M, Agrawal R, Deorari AK et al. Sepsis in newborn. Indian J Pediatr 2008: 75: 261-66. 
Y. R. Khinchi et al, Profile of Neonatal sepsis

Table-1: Profile of Neonatal sepsis:

\begin{tabular}{llll}
\hline Feature & Inborn & Out born & Total (215) \\
& $(40)$ & $(175)$ & $(\%)$ \\
\hline Refusal to feeds & $28(70 \%)$ & $132(75 \%)$ & $160(74 \%)$ \\
Poor activity and Cry & $21(52 \%)$ & $67(38 \%)$ & $88(41 \%)$ \\
Tachypnea / Respiratory distress & $32(80 \%)$ & $129(73 \%)$ & $161(75 \%)$ \\
Fever & $26(65 \%)$ & $122(69 \%)$ & $148(69 \%)$ \\
Jaundice & $28(70 \%)$ & $59(33 \%)$ & $87(40 \%)$ \\
Apnea & $9(22 \%)$ & $24(13 \%)$ & $33(15 \%)$ \\
Hypothermia & $2(5 \%)$ & $23(13 \%)$ & $25(11.6 \%)$ \\
Cyanosis & $12(30 \%)$ & $78(44.5 \%)$ & $90(41 \%)$ \\
CRT(Capillary Refill Time) $>$ 3 Sec & $9(22.5 \%)$ & $82(46.8 \%)$ & $91(42.3 \%)$ \\
Bleeding/ Patechie/ Purpura & $12(30 \%)$ & $36(20.5 \%)$ & $48(22.3 \%)$ \\
Convulsions & $8(20 \%)$ & $36(20.5 \%)$ & $44(20.4 \%)$ \\
Umbilical sepsis / Skin infections & $2(5 \%)$ & $31(17.7 \%)$ & $33(15.3 \%)$ \\
Chest X-Ray suggestive of Respiratory infection & $24(60 \%)$ & $68(38.8 \%)$ & $92(42.7 \%)$ \\
Clinical and chest X-Ray with empyema & - & $1(0.57 \%)$ & $1(0.46 \%)$ \\
Positive septic screen (CRP/CBC/PBF) & $26(65 \%)$ & $109(62.2 \%)$ & $135(62.7 \%)$ \\
Positive blood culture (out of 22 samples sent) & - & $1(0.57 \%)$ & $1(0.46 \%)$ \\
Meningitis (CSF: Cell count/Gram stain/Biochem.) & $5(12.5 \%)$ & $28(16 \%)$ & $33(15.3 \%)$ \\
CT scan showing complications (Meningitis cases) & - & $2(1.14 \%)$ & $2(0.93 \%)$ \\
Mortality due to Sepsis & $3(7.5 \%)$ & $19(11 \%)$ & $22(10.2 \%)$ \\
\hline
\end{tabular}

Table-2: Distribution of cases according to sex and septic/ non-septic:

\begin{tabular}{llll}
\hline Sex & Septic & Non-septic & Total \\
\hline Male & $140(65.1 \%)$ & $131(66.8 \%)$ & 271 \\
Female & $75(34.9 \%)$ & $65(33.1 \%)$ & 140 \\
Total & 215 & 196 & 411 \\
\hline
\end{tabular}

( $\mathrm{p}$ value $<0.05)$

Table-3: Distribution of cases according to place of delivery:

\begin{tabular}{llll}
\hline Sepsis cases & Septic & Non-septic & Total \\
\hline Out born (Extramural) & $175(59 \%)$ & $122(41 \%)$ & 297 \\
Inborn (Intramural) & $40(35 \%)$ & $74(65 \%)$ & 114 \\
Total & $215(52.3 \%)$ & $196(47.6 \%)$ & 411 \\
\hline
\end{tabular}

( $\mathrm{p}$ value $<0.05)$ 
Table-4: Distribution of sepsis cases according to onset of Neonatal sepsis:

\begin{tabular}{llll}
\hline Place of delivery & Early onset sepsis & Late onset sepsis & Total \\
\hline Inborn (intramural) & $32(80 \%)$ & $8(20 \%)$ & 40 \\
Out born (extramural) & $99(56 \%)$ & $76(44 \%)$ & 175 \\
Total & $131(60.9 \%)$ & $84(39 \%)$ & 215 \\
\hline
\end{tabular}

( $\mathrm{p}$ value $<0.05$ )

Table-5: Neonatal sepsis according to birth weight:

\begin{tabular}{llll}
\hline Weight group & Septic & Non septic & Total \\
\hline $2.5 \mathrm{Kg}$ or more & $102(42.6 \%)$ & $137(57.3 \%)$ & 239 \\
Low birth weight & $83(64.3 \%)$ & $46(35.6 \%)$ & 129 \\
$(1.5 \mathrm{Kg}$ to $<2.5 \mathrm{Kg})$ & & & \\
Very/extremely low birth weight & $30(69.7 \%)$ & $13(30.2 \%)$ & 43 \\
$(<1.5 \mathrm{Kg})$ & & & \\
All weight group & $215(52.3 \%)$ & $196(47.6)$ & 411 \\
\hline
\end{tabular}

( $\mathrm{p}$ value $<0.000)$ 